\title{
A finite element model of the electrofusion welding of thermoplastic pipes
}

\author{
G F Rosala ${ }^{1}$, A J Day ${ }^{1}$ and A S Wood ${ }^{2}$ \\ ${ }^{1}$ Department of Mechanical and Manufacturing Engineering, University of Bradford, West Yorkshire \\ ${ }^{2}$ Department of Mathematics, University of Bradford, West Yorkshire
}

\begin{abstract}
An advanced finite element (FE) model of the electrofusion welding of thermoplastic pipes has been developed using the ABAQUS FE package. The heat transfer analysis is coupled with thermal deformation analysis to include the time-dependent closure of the initial gap between the pipe and fitting. The effect of radial melt movement into the interface is modelled using a new 'virtual material movement' technique. The predicted results (temperature distribution in the weld region, melt affected zones and gap closure time) are compared with experimental data and good agreement is found.
\end{abstract}

Keywords: finite element model, electrofusion welding, thermoplastic pipes, heat transfer modelling, polyethylene

\section{NOTATION \\ $d \quad$ fitting-pipe interface thickness \\ $f_{q} \quad$ heat flux through the fitting-pipe interface \\ $h \quad$ gap thermal conductance \\ $k$ thermal conductivity \\ $l \quad$ wire length \\ $P_{\mathrm{v}} \quad$ unit volumetric power input \\ $R_{\Omega} \quad$ wire unit ohmic resistance \\ SDR standard diameter ratio (ratio of the pipe out- side diameter to the wall thickness) \\ $T, \Delta T$ temperature and temperature variation \\ $U \quad$ voltage \\ $v \quad$ specific volume \\ $\alpha \quad$ coefficient of thermal expansion \\ $\alpha_{\gamma} \quad$ temperature coefficient of electrical resistance \\ $\omega \quad$ wire cross-sectional area}

\section{INTRODUCTION}

Over the past three decades the use of thermoplastic pipes has emerged as the preferred choice for large utilities operating water and gas distribution networks, mainly because of their freedom from attack by corrosion and the ability to easily form a fully fused, strong and leakproof joint (1). Various welding techniques with a good record of field performance are used, including socket, butt and electrofusion welding (EFW). The

The MS was received on 14 December 1995 and was accepted for publication on 9 July 1996. latter is the preferred jointing system for polyethylene (PE) pipes where the available space and pipe movement are limited and cross-fusion of polyethylene resins is needed.

The basic principle of the EFW process is that an electrofusion fitting, with its built-in conductive winding, is connected to a power supply and heat, which is generated in the wire by the Joule effect, diffuses into the surrounding polymer, leading to a progressive melting of the fitting and pipe contact surfaces. After cooling, a sound bonding of the pipe and fitting is obtained due to the physicochemical mechanism of polymer welding (2).

The fabrication of a good EFW joint, assessed in terms of its ability to withstand the service loads for a long period of time (a minimum of 50 years according to present practice), is dependent upon the correct choice of the many possible combinations of design and process parameters, such as temperature of the fitting-pipe interface, heating-cooling rates, time (process duration), contact pressure during heating and cooling, fitting and pipe resins' weldability and contamination state prior to welding. Although the allowable range of these parameters can be fixed by employing a trial-and-error approach, the benefits of a predictive model that can be used to simulate different welding conditions are clear. Since 1985 much research work has been undertaken in the United Kingdom, France, Japan and the United States towards the development of suitable modelling techniques. The finite difference method was used to develop one-dimensional heat transfer models, with or without inclusion of the effects of the initial gap between the pipe and fitting and the latent heat of fusion $(3,4)$. 
Several finite element (FE) packages were also employed to model the EFW process, such as CASTEM, developed by Kobe Steel Limited, Japan, ALI-BABA, developed by Direction des Etudes et Recherches d'Electricite de France, and ABAQUS, developed by Hibbit, Karlsson and Sorensen, United States (4-7). A good review of the published EFW modelling work can be found in reference $(\mathbf{8})$.

However, all of the EFW models developed so far are based on a continuum solid approach and do not take into consideration the effect of radial melt movement into the initial gap between the fitting and pipe. This becomes increasingly important for larger size fittings. This paper describes how an EFW finite element model can be refined to include this effect by using an original 'virtual material movement' (VMM) method.

\section{THE FINITE ELEMENT MODEL}

\subsection{Problem definition and modelling strategy}

The problem to be modelled is one of a transient heat transfer analysis coupled with a thermomechanical stress analysis. In the transient heat transfer formulation there are two consecutive changes of phase (melting and re-solidification of the polymer) in the fitting and pipe domains and a variable thermal resistance between them. The thermomechanical stress analysis (in which any manufacture-induced residual stresses in the pipe and fitting have not been considered) has to model two cylindrical bodies (fitting and pipe), which expand and distort developing thermal stresses and temperature variation through the wall thickness radially and axially. After the initial gap between the fitting and pipe closes, contact stresses are generated. During the cooling period the bodies, at this stage welded over a part of their contact surfaces, try to recover their initial shape and dimension, minus the plastic deformations. Thus the stress distribution changes and at the end of the cooling period when the temperature field is again uniform over the whole domain a residual stress state can be expected.

The presence of the initial fitting-pipe gap is an important modelling issue, mainly because the gap constitutes a barrier to the heat flowing from the fitting to the pipe. Moreover, the uneven distribution of the initial gap in the axial and circumferential directions is the main factor determining the extent of radial melt movement (8). The radial melt movement is responsible for the variable relative position of the contact interface and heating element during the EFW process, irrespective of the initial configuration of the embedded wire. Thus in terms of modelling the heating period of the EFW process, an ability to describe an average movement of the wire and interface in opposite radial directions becomes essential.

The very low absolute value of the melt movement in a standard EFW process does not justify the use of a flow-based model. However, a model built on a solid continuum approach, in which the molten regions of the joint are treated as a 'soft solid', is appropriate if the effect of the radial melt movement can be included. The finite element package ABAQUS (9) was chosen because it accommodates all the main features required by the EFW process, including interface-type elements for describing pipe-fitting interaction, temperaturedependent material properties, phase change and coupled thermal-displacement analysis. ABAQUS also allows the user to develop dedicated interface heat transfer subroutines.

\subsection{Domain discretization, time increment selection and analysis accuracy}

The transient heat transfer occurring in the joint during the heating period is a diffusion-dominated process. Therefore, in response to the sudden application of the electric current the maximum increase in temperature occurs initially in the vicinity of the wires. In any transient problem the time increment and the element size in the direction of the disturbance propagation are related. A minimum time increment, given as a function of the material's thermal diffusivity and the 'next to disturbance' element dimension in the direction of the temperature gradient, is recommended in ABAQUS. If the analysis requires an accurate capture of the temperature evolution in regions where high thermal gradients are expected, then a suitably refined spatial mesh has to be used. Consequently, the FE mesh was graded to provide finer discretization near the fitting-pipe interface where the thermal conditions change rapidly. This can be seen in Fig. 1, which represents an axisymmetric model for one half of a $125 \mathrm{~mm}$ EFW coupler and the corresponding SDR 11 pipe. The aspect ratio of all elements is maintained within the ABAQUS recommended limit of $10: 1$.

In ABAQUS the maximum temperature change allowed in any time increment, which controls the accuracy to which the transient analysis is integrated in time, can be selected. After the first time step, an automatic time stepping routine (having an in-built logic to accommodate the expected exponential decay or growth characteristic to transient heat transfer analysis) can be invoked which attempts to increase the time step to reduce computation time.

\subsection{Element selection}

For a coupled temperature-displacement analysis ABAQUS provides continuum elements with both temperature and displacement degrees of freedom. These elements have either linear (first-order) or quadratic (second-order) interpolation for displacements but the temperature is always linearly interpolated. From this it 


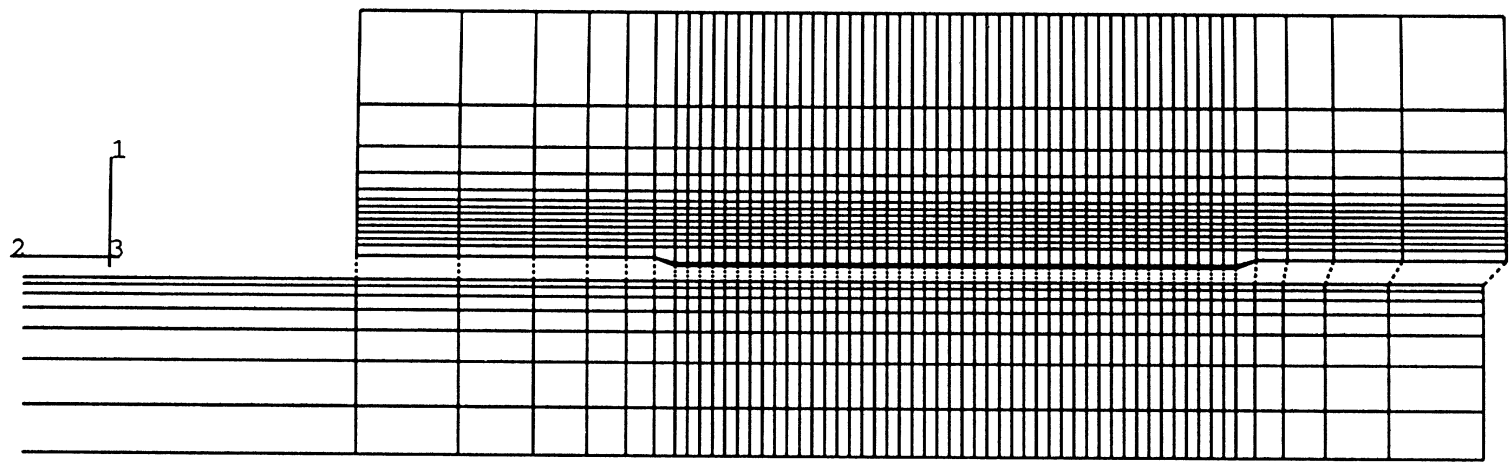

Fig. 1 Finite element mesh for an axisymmetric EFW model (125 mm coupler/SDR 11 pipe)

is clear that temperature gradient discontinuities such as those occurring in a change of phase are not allowed within the elements; therefore the mesh has to be refined where phase changes are expected. Although the quadratic displacement interpolation elements respond well to any bending effects occurring in the analysis, they are not suited to the analysis of non-smooth non-linearities such as latent heat effects (9). Elements with linear displacement interpolation were selected for this analysis despite the disadvantages of a stiff response in bending and a lower thermal accuracy (the thermal strain is taken as constant over the element for the sake of mechanical and thermal strain compatibility), because these limitations could be overcome by refining the mesh. Interface (gap) elements with both displacement and temperature degrees of freedom were used to link the fitting and the pipe domains. The interface elements provide the transmission of forces between the bodies in contact, and the way in which these forces are transmitted depends upon the surface theory applied, e.g. to allow no separation once the contact has been established, or some overclosure before contact constraints are imposed. In this analysis the 'no separation' theory was employed, in order to improve the solvability by preventing any oscillatory contact between the pipe and the fitting. Although this approach involved a certain approximation, it was considered that any error introduced could be monitored through the examination of the forces developed in the interface elements. Additionally, the gap elements have no thickness and no associated 'thermal mass' (i.e. no thermal energy is stored in the interface), but allow different temperatures on their two sides. Heat may flow across the interface via conduction and radiation: calculations made during the development of the model indicated that during gap closure approximately 20 per cent of the heat energy would be transferred from the fitting to the pipe by radiation and 80 per cent by conduction. The gap closure stage typically accounts for less than 25 per cent of the total heat energy transmitted from the fitting to the pipe, so that radiation heat transfer accounts for a maximum of 5 per cent of the total heat transfer during the complete EFW process. Radiation heat transfer has therefore not been included in the model. Because conduction heat transfer occurs only if a form of matter exists within the gap it is considered to be proportional to the conductivity of the material within the interface and the temperature difference between the surfaces, given by the expression:

$$
f_{q}=h\left(T_{\mathrm{A}}-T_{\mathrm{B}}\right)
$$

where $h=k / d$ and $T_{\mathrm{A}}$ and $T_{\mathrm{B}}$ are the temperatures of the two surfaces. As the gap clearance approaches zero there is no interface resistance to restrict heat transfer across the gap and therefore the conductance approaches infinity. In the model, a suitably high finite value was used to describe the interface thermal properties in this situation.

\subsection{The VMM method}

The standard approach to analysing the heat transfer across the gap, as described in Section 2.3, has proved unsatisfactory for an accurate description of the gap closure process occurring in the electrofusion welding of thermoplastic pipes. It has been shown by experiment (8) that, if there were a clearance between the fitting and the pipe at the beginning of the EFW process, then a radial melt movement (from the fitting to the pipe) would be generated during the heating period. Effectively the wires remain close to their original position while the polymer melt flows between them to the interface. The consequence of this movement is that, whatever the initial wire embedding configuration, a variable distance between the heating element and the bonding interface will occur. This is a very important observation which should be appropriately treated by the modelling. The standard modelling approach starts from the initial condition shown in Fig. $2 \mathrm{a}$ and assumes the final condition to be that shown in Fig. 2 b, but in reality the condition is that illustrated in Fig. 2c.

Because the 'soft-solid' approach was adopted, flow effects could not be modelled. Consequently, the VMM method was devised to simulate the effects of melt movement into the fitting-pipe gap, between the heating 


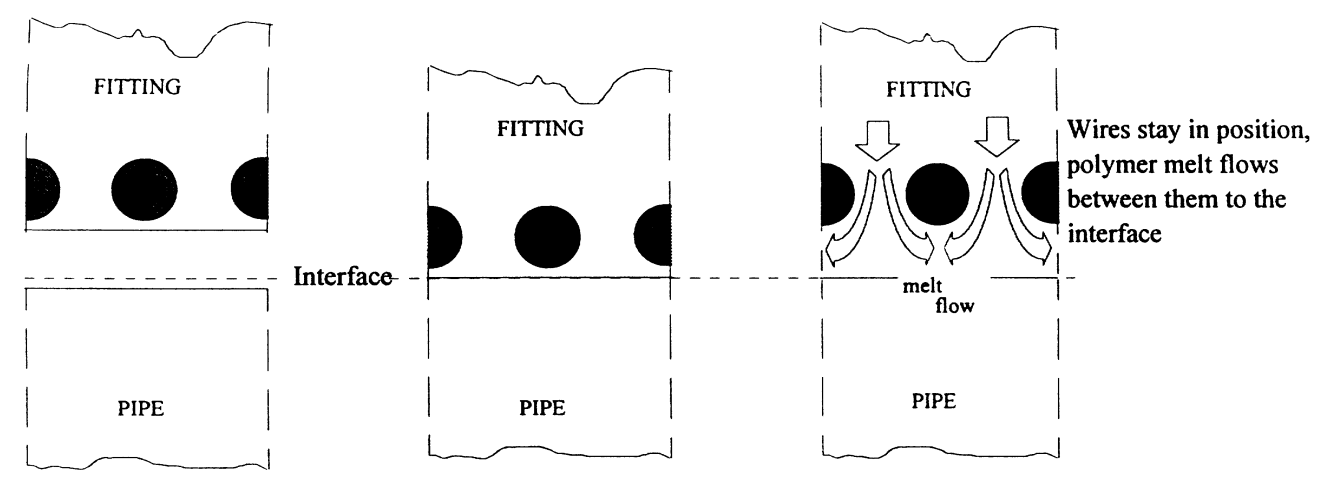

(a) Initial gap status

(b) Standard gap closure model

(c) Real gap closure situation

Fig. 2 Fitting-pipe gap closure models

element and the interface. The basis of the VMM method is that at any increment in time, before the gap closes, the fitting-pipe interface is composed of two layers: air, with a thickness equal to that of the current value of the gap, and melt from the fitting material. The thickness of the melt layer is calculated from the variation of radius of the fitting bore, and a composite gap conductance, which leads to a correct fitting-pipe heat transfer formulation, can then be computed. The thermal resistance $(1 / h)$ of a composite domain can be computed as the sum of the resistances of individual layers:

$$
\frac{1}{h}=\frac{1}{h_{\text {air }}}+\frac{1}{h_{\mathrm{PE}}}
$$

where $1 / h_{\text {air }}$ and $1 / h_{\mathrm{PE}}$ represent the thermal resistance of the air and melt layers respectively. Rearranging equation (2) gives

$$
h=\frac{h_{\mathrm{air}} h_{\mathrm{PE}}}{h_{\mathrm{air}}+h_{\mathrm{PE}}}
$$

and substituting $h_{\mathrm{i}}=k_{\mathrm{i}} / d_{\mathrm{i}}$ gives the gap conductance

$$
h=\frac{k_{\mathrm{air}} k_{\mathrm{PE}}}{k_{\mathrm{air}} d_{\mathrm{PE}}+k_{\mathrm{PE}} d_{\mathrm{air}}}
$$

where $d_{\mathrm{air}}$ and $d_{\mathrm{PE}}$ represent the air and melt layers' thicknesses respectively, while $k_{\mathrm{air}}$ and $k_{\mathrm{PE}}$ stand for the material thermal conductivities.

For an initial fitting-pipe gap of $1 \mathrm{~mm}$ and the participation of the molten fitting material in the closure of the initial gap estimated at roughly 85 per cent [an acceptable approximation in the light of the pipe bore measurement results (8)], the variation of $h_{\mathrm{air}}, h_{\mathrm{PE}}$ and $h$ is shown in Fig. 3. It can be seen that, although the presence of the melt in the interface does not affect the first $0.6 \mathrm{~mm}$ of the closure, it later significantly affects the heat transfer conditions, which illustrates the importance of modelling the radial melt movement. The VMM method was incorporated through a user subroutine.

\subsection{Material properties}

Polymer conductivity and specific heat were both described as temperature dependent by giving conductivity and specific heat at several different temperatures. Only two different values were required for the polyethylene specific heat, corresponding to the solid and liquid phases, since ABAQUS uses a special latent heat routine that avoids the use of a temperature-dependent description of the specific heat (see Table 1). In this routine the latent heat is considered to be absorbed over a temperature range, between a lower (solidus) temperature and an upper (liquidus) temperature, and liberated over the same range when cooling. However, since semicrystalline polymers melt and re-solidify over different temperature ranges, absorbing and liberating different quantities of thermal energy, average values were used (see Table 2). Tables 1 and 2 refer to two different polyethylene materials: Finathene 3802B was the pipe material and Rigidex PC002-50 was the fitting material studied here.

In ABAQUS the material density cannot be given as a function of temperature. However, because material density and specific heat always appear as a product in the heat transfer governing equations, the variation of density with temperature was incorporated into the value of the specific heat.

A good description of the variation of the coefficient of thermal expansion with temperature, including expansion due to a change of phase, was an essential feature for the model in terms of gap closure prediction and thermal stress patterns. Specific volume versus temperature and pressure curves ( $p v T$ diagrams) may be used to determine the variation of the thermal expansion coefficient. Assuming that the material is isotropic, the value of the coefficient of thermal expansion, including the change in volume due to melting or re-crystallization, can be calculated from

$$
\alpha \cong \frac{v_{T_{2}}-v_{T_{1}}}{3 v_{T_{1}} \Delta T}
$$




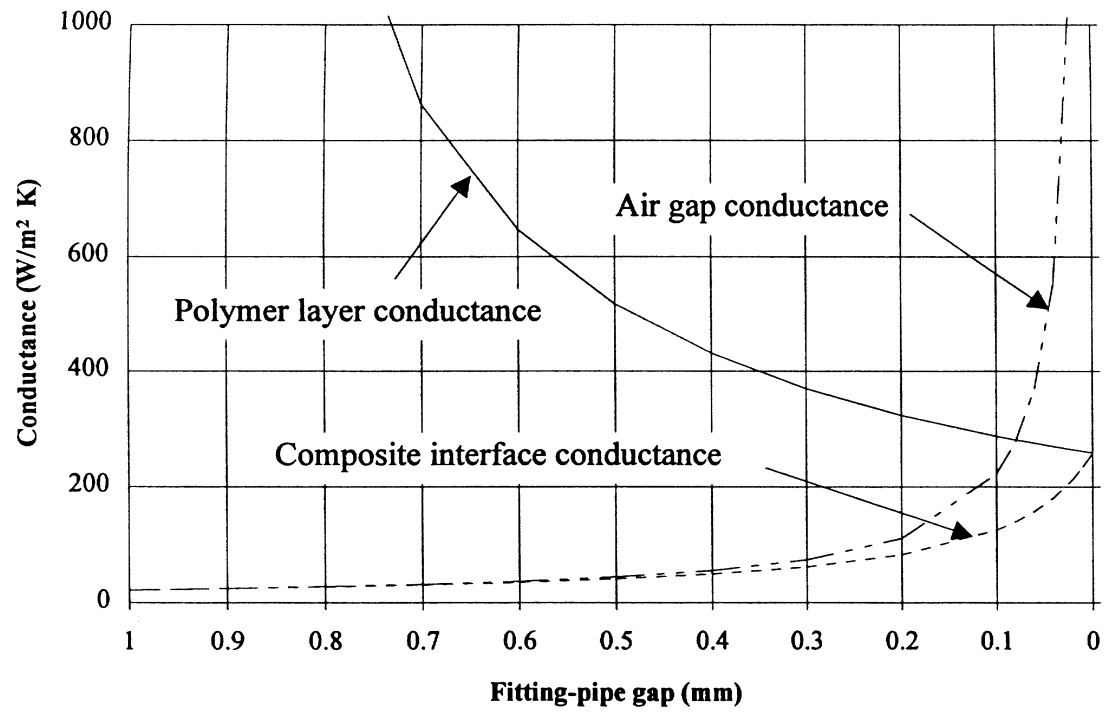

Fig. 3 Interface conductance dependence on gap closure

Table 1 Temperature-dependent thermal properties of two medium density polyethylenes

\begin{tabular}{|c|c|c|c|c|}
\hline \multirow[b]{2}{*}{ Temperature $\left({ }^{\circ} \mathrm{C}\right)$} & \multicolumn{2}{|c|}{$\begin{array}{l}\text { Thermal conductivity } \\
(\mathrm{W} / \mathrm{m} \mathrm{K})\end{array}$} & \multicolumn{2}{|c|}{$\begin{array}{l}\text { Specific heat capacity } \\
(\mathrm{J} / \mathrm{kg} \mathrm{K})\end{array}$} \\
\hline & Finathene $3802 \mathrm{~B}$ & Rigidex PC002-50 & Finathene 3802B & Rigidex PC002-50 \\
\hline 25 & 0.40 & 0.39 & $2.01 \times 10^{3}$ & $2.40 \times 10^{3}$ \\
\hline 100 & 0.31 & 0.32 & - & - \\
\hline 125 & 0.27 & 0.29 & - & - \\
\hline 160 & 0.21 & 0.26 & $2.60 \times 10^{3}$ & $3.10 \times 10^{3}$ \\
\hline
\end{tabular}

Table 2 Average latent heat values used in the analysis

\begin{tabular}{lcc}
\hline \multirow{2}{*}{ Parameter } & \multicolumn{2}{c}{ Polyethylene type } \\
\cline { 2 - 3 } & Finathene 3802B & Rigidex PC002-50 \\
\hline Temperature range $\left({ }^{\circ} \mathrm{C}\right)$ & $112-122$ & $114-122$ \\
Latent heat $(\mathrm{J} / \mathrm{kg})$ & $144.2 \times 10^{3}$ & $150.5 \times 10^{3}$ \\
\hline
\end{tabular}

where $v_{T_{2}}$ and $v_{T_{1}}$ represent specific volume values at temperatures $T_{1}$ and $T_{2}$ respectively (see Table 3 ).

The thermal conductivity and the specific heat of the electric wire are typically $71 \mathrm{~W} / \mathrm{m} \mathrm{K}$ and $456 \mathrm{~J} / \mathrm{kg} \mathrm{K}$ for a 2 per cent manganese-nickel alloy and $130 \mathrm{~W} / \mathrm{m} \mathrm{K}$ and $380 \mathrm{~J} / \mathrm{kg} \mathrm{K}$ for a 99.9 per cent copper material. The density and thermal expansion coefficient of the wire do not vary significantly with temperature over the range associ-

Table 3 Temperature-dependent thermal expansion behaviour of polyethylene, including the change of phase

\begin{tabular}{cc}
\hline Temperature $\left({ }^{\circ} \mathrm{C}\right)$ & Coefficient of thermal expansion $(1 / \mathrm{K})$ \\
\hline 100 & $2.55 \times 10^{-4}$ \\
130 & $4.71 \times 10^{-4}$ \\
150 & $4.35 \times 10^{-4}$ \\
280 & $3.29 \times 10^{-4}$ \\
\hline
\end{tabular}

E03995 (C) IMechE 1997 ated with the EFW process, and the most common values given to them are $8.85 \times 10^{3} \mathrm{~kg} / \mathrm{m}^{3}$ and $17.5 \times 10^{-6} \mathrm{~mm} / \mathrm{K}$ for density and thermal expansion respectively $(\mathbf{1 0})$.

Because in this research the stress problem has been treated in a 'qualitative' way, with the aim of the search being more for patterns than for values, the creep and stress relaxation effects have not been considered. Thus, a temperature-dependent elastic-plastic hardening model was employed to describe the 'immediate' response of the solid polymer. The equivalent pressure stress independent yield model in ABAQUS is a classic Mises model, in which true stress-strain data are used to provide isotropic hardening values. The elastic properties of the material were defined by Young's modulus as a function of temperature and by Poisson's ratio $(\mathbf{8})$ :

$$
\begin{array}{ll}
\text { Young's modulus } & 362 \mathrm{MPa} \text { at } 25^{\circ} \mathrm{C} \text { to } 34 \mathrm{MPa} \\
& \text { at } 110{ }^{\circ} \mathrm{C} \\
\text { Yield stress } & 14 \mathrm{MPa} \text { at } 25^{\circ} \mathrm{C} \text { to } 2.3 \mathrm{MPa} \\
& \text { at } 110^{\circ} \mathrm{C} \\
\text { Poisson's ratio } & 0.45
\end{array}
$$

As the polymer melts, its capacity to withstand external loads decreases to zero and if there is a free boundary 
it will flow. However, in the EFW process, the melted polymer is contained in an 'envelope' between the pipe and fitting, which prevents this flow. Thus the melt pool was considered to be a 'soft solid' responding elastically to the loading.

Constant values of $2.11 \times 10^{5} \mathrm{MPa}$ and 0.3 , for the wire elastic moduli and Poisson's ratio respectively, were initially considered appropriate in the analysis. However, it was found that this description not only introduced errors into the fitting deformation prediction but also created convergence problems. This was because the wire and the surrounding polymer move in opposite radial directions; as the very stiff elements associated with the wire try to move outwards, following their thermal expansion, they prevent the neighbouring polymer elements from moving inwards to satisfy equilibrium in the soft solid domain. To overcome this the wire material was softened by being given the same mechanical properties as the polymer. In this way the wires did not act as a restraint to the natural deformation trend of the fitting polymeric material. This simplification is justified since, in real life, the very hot wires do not oppose the movement of the melt.

\subsection{Loading procedure}

From the point of view of loading, the EFW process involves a two-step analysis: the first corresponds to the time the electric power is on (the heating period) and the second when the power supply is removed (the cooling period). As a result of the power input being temperature dependent (the wire material resistivity can be described as a linear function of temperature) a non-uniform body flux was calculated from

$$
P_{\mathrm{v}}=\frac{U^{2}}{R_{\Omega 0} \omega_{0} l_{0}^{2}\left(1+\alpha_{\gamma} \Delta T\right)(1+\alpha \Delta T)^{2}}
$$

where the subscript 0 refers to room temperature values.

\subsection{Initial and boundary conditions}

For a transient heat transfer and thermal stress problem, initial conditions refer to the initial temperature at which transient heat transfer is supposed to start and the domain of investigation is considered as a stress-free continuum. To allow an easier correlation with the experiments this value was set to equal the laboratory ambient temperature.

Thermal boundary conditions were employed to describe a linear heat transfer at the pipe and fitting surfaces into an environment assumed to have a constant temperature. The procedure defines the heat lost from the pipe and fitting to be dependent upon a 'film conductance value', given as an input. The implemented value of these coefficients was $4.2 \mathrm{~W} / \mathrm{m}^{2} \mathrm{~K}$ for the pipe bore and $8 \mathrm{~W} / \mathrm{m}^{2} \mathrm{~K}$ for the fitting outer surface. They match the real cooling conditions of the associated experimen- tal work and are also close to the values used by other researchers $(\mathbf{6}, \mathbf{8})$. Model restraints were defined through kinematic boundary conditions, implemented by suppressing the appropriate degrees of freedom.

\section{RESULTS AND DISCUSSION}

\subsection{Effect of the gap modelling method on results}

To demonstrate the importance of the correct modelling of the initial fitting-pipe clearance, three separate heat transfer analyses were executed for a joint consisting of a $125 \mathrm{~mm}$ coupler and an SDR 17.6 pipe, using three different gap models, as described below:

(a) no initial clearance, with a fixed value of the interface conductance;

(b) initial radial clearance of $1 \mathrm{~mm}$, simulated gap closure time of $80 \mathrm{~s}$, gap conductance given by the standard model (see Fig. 2);

(c) initial radial clearance of $1 \mathrm{~mm}$, simulated gap closure time of $80 \mathrm{~s}$, gap conductance given by the VMM method.

The results of this investigation are presented in Figs 4 and 5. It can be seen from Fig. 4 that both the "no gap' and 'standard gap' models predict nearly the same interface temperature at the end of the heating period. However, when modelling the melt movement into the gap a substantially lower value is predicted (some $20^{\circ} \mathrm{C}$ for this simulation). It can also be seen that, although the effect of gap modelling is not relevant for the fitting outer surface temperature history, it does change the evolution of the pipe bore temperature. The predicted value of the melt layer thickness at the end of the heating period, in the fitting and pipe domains, can be computed from the diagram shown in Fig. 5 (by measuring the distances from the interface to points situated at the intersection of the diagram with an isothermal line at melt temperature). It can be seen that the 'no gap' model predicts pipe and fitting melt thickness values some 40 per cent larger and 10 per cent smaller than the VMM gap model respectively, assuming a melt temperature of $125^{\circ} \mathrm{C}$. The analysis using the standard model gives melt thickness results between the previous two, closer to those predicted using the gap model based on the VMM method. Experimental validation of these results is discussed next in Section 3.2.

These investigations showed that the initial fitting-pipe clearance is an essential issue for modelling the EFW process and accurate results, in terms of interface temperature and melt thickness value, can only be achieved by considering the radial melt movement into the interface, achieved here by the VMM method.

E03995 (C) IMechE 1997 


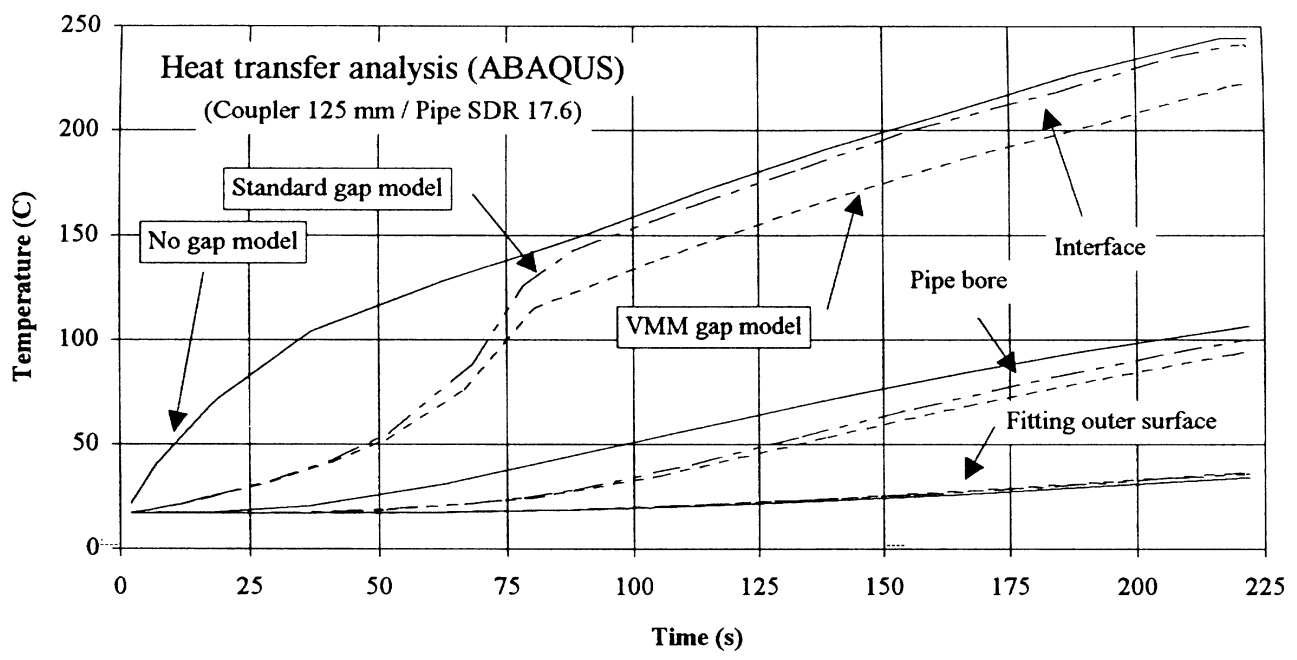

Fig. 4 Predicted temperature histories through the EFW heating period

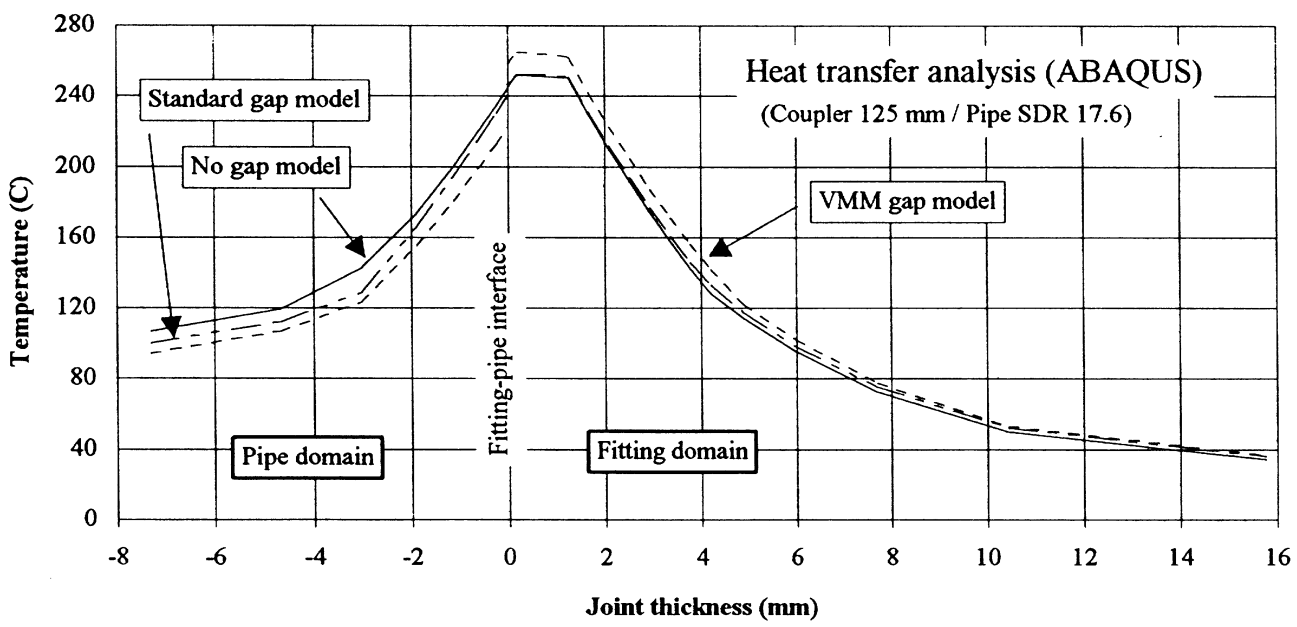

Fig. 5 Predicted temperature distribution across the joint thickness (at the end of the heating period)

\subsection{Validation of the EFW model}

Several simulations of an EFW process, involving a $125 \mathrm{~mm}$ coupler and an SDR 11 pipe, were performed by running fully coupled temperature-displacement analyses over the heating and cooling periods, up to 2400 s. Predicted results concerning temperature distributions and thermal distortion are compared with measured values, generated through the welding of instrumented pipes and fittings $(\mathbf{8})$. The predicted extent of the melt in the fitting and pipe is compared with results from associated microstructural studies (11).

Four critical regions are defined within an EFW joint relating to the temperature history, viz. the fitting-pipe interface, the immediate vicinity of the wire, the pipe bore and the fitting outer surface. The fitting-pipe interface temperature is one of the predominant param- eters for joint strength evaluation. Welding occurs as the hot melt front advances radially inwards from the fitting bore, to meet the pipe surface. The following discussion concentrates on the interface temperature values near the centre of the fusion zone length at two positions, one in a radial plane through the centre of the middle wire and the other in a radial plane positioned one half-pitch distance away. Throughout the EFW process the polymer in contact with the wire reaches the highest temperature. If the polymer is overheated (usually above $350^{\circ} \mathrm{C}$ ) it becomes thermally degraded, which shows up as a decrease in molecular weight and mechanical properties. The temperature of the pipe bore and fitting outer surface reach their maximum values in the centre of the fusion zone length, during the cooling period. These maximum temperatures control the maximum amount of energy that can be put into a welding process without 


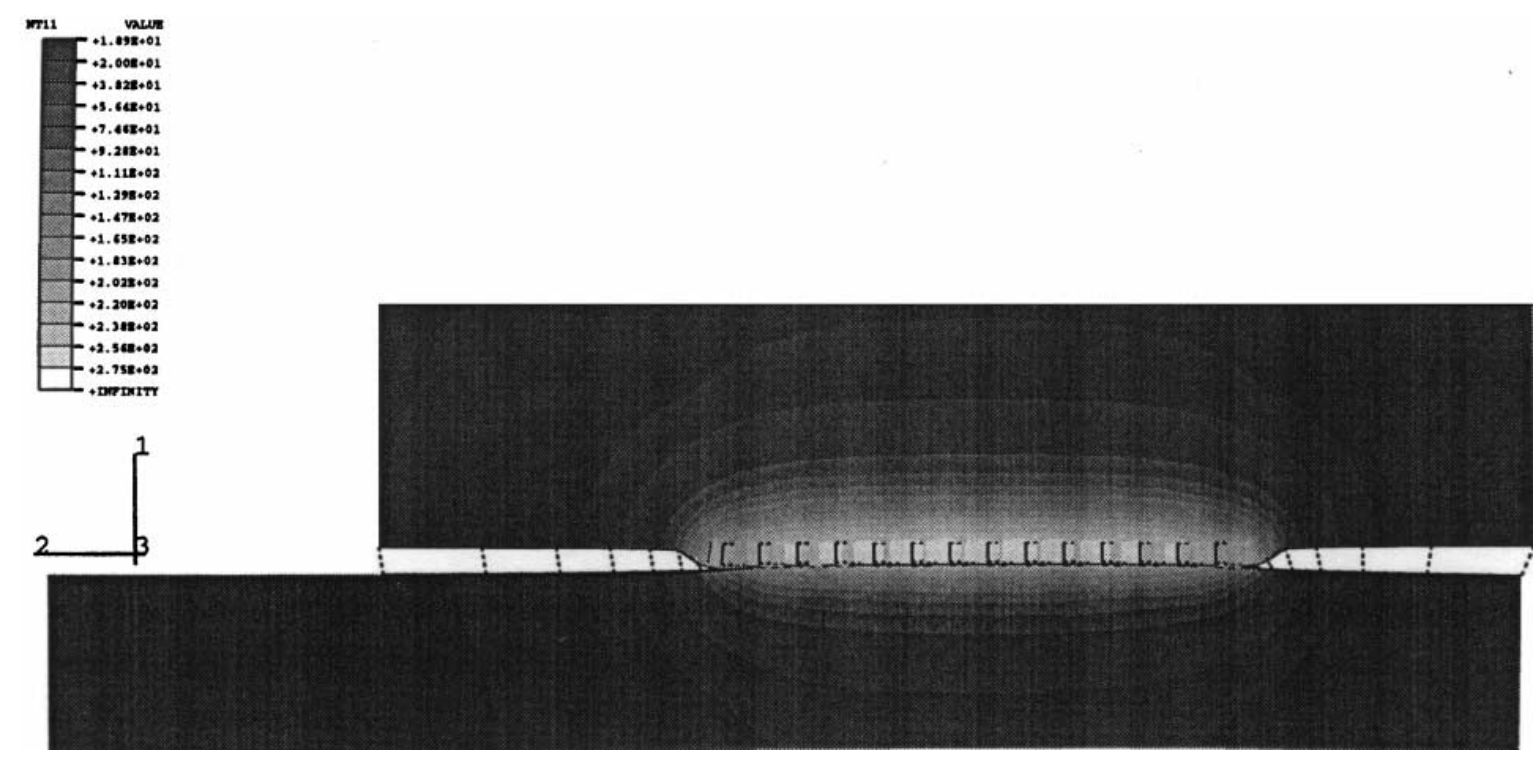

Fig. 6 Electrofusion weld simulation (temperature distribution and thermal distortion in the joint)

leading to the collapse of the joint through excessive softness due to an exaggerated melt depth.

Contour plots of the temperature distribution in the fitting and pipe domains and the associated thermal distortions are presented in Fig. 6. To simplify the comparison between the predicted and measured values of temperature through the EFW process, Fig. 7 shows the variation of the interface and wire temperatures by superimposing the predicted values over the experimental values for the heating period and Fig. 8 shows the variation of the interface and wire temperatures for the whole heating-cooling period. These indicate the maximum values of the pipe bore and fitting outer surface temperatures and validate the surface cooling coefficients used within the models (Fig. 8). Regarding the maximum interface temperature at the end of the heating period good agreement can be found between the predicted values and the experimental data. Good consistency is also found for the maximum temperature of the wire, although the predicted wire temperature history differs slightly from the experimental curve. This is because the experimental curves, computed from the variation of the electric current over the whole coil, cannot detect local variations in temperature, such as those occurring when the gap closes. The predicted temperature history of the fitting outer surface is in very good agreement with the experimental data. The pipe bore temperature history and maximum temperature

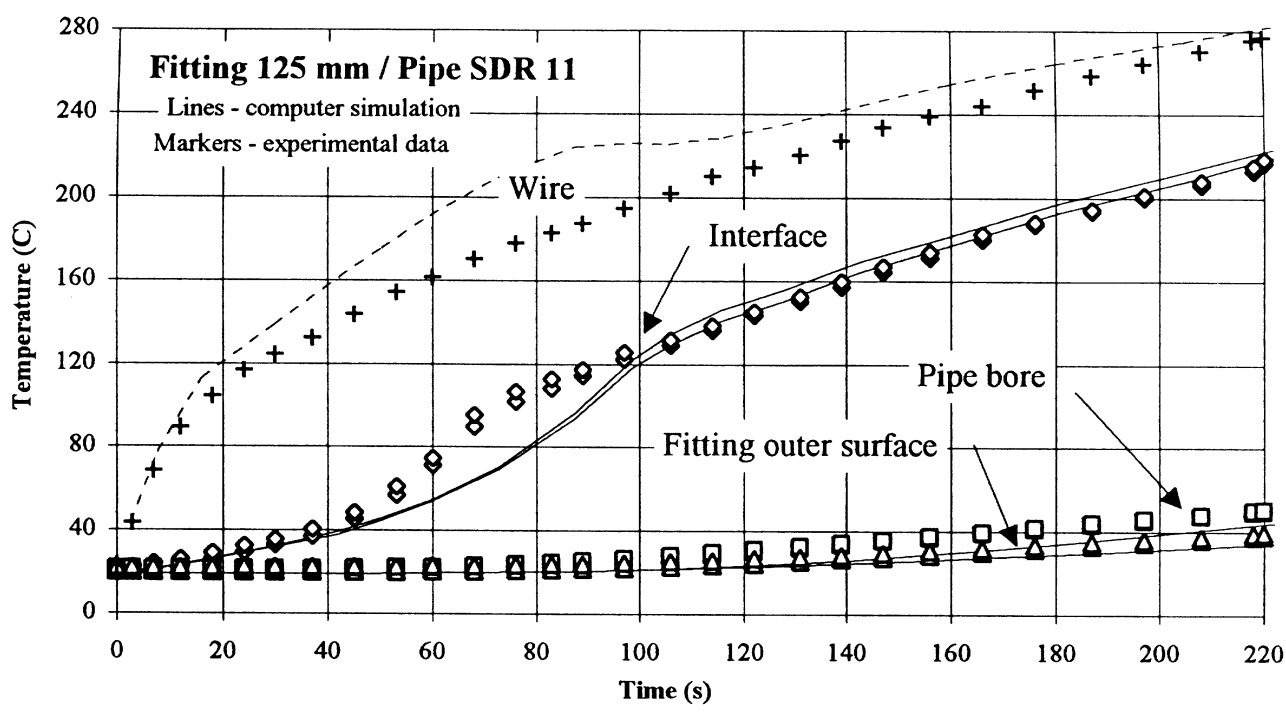

Fig. 7 Correlation between prediction and experimental data (heating period only) 


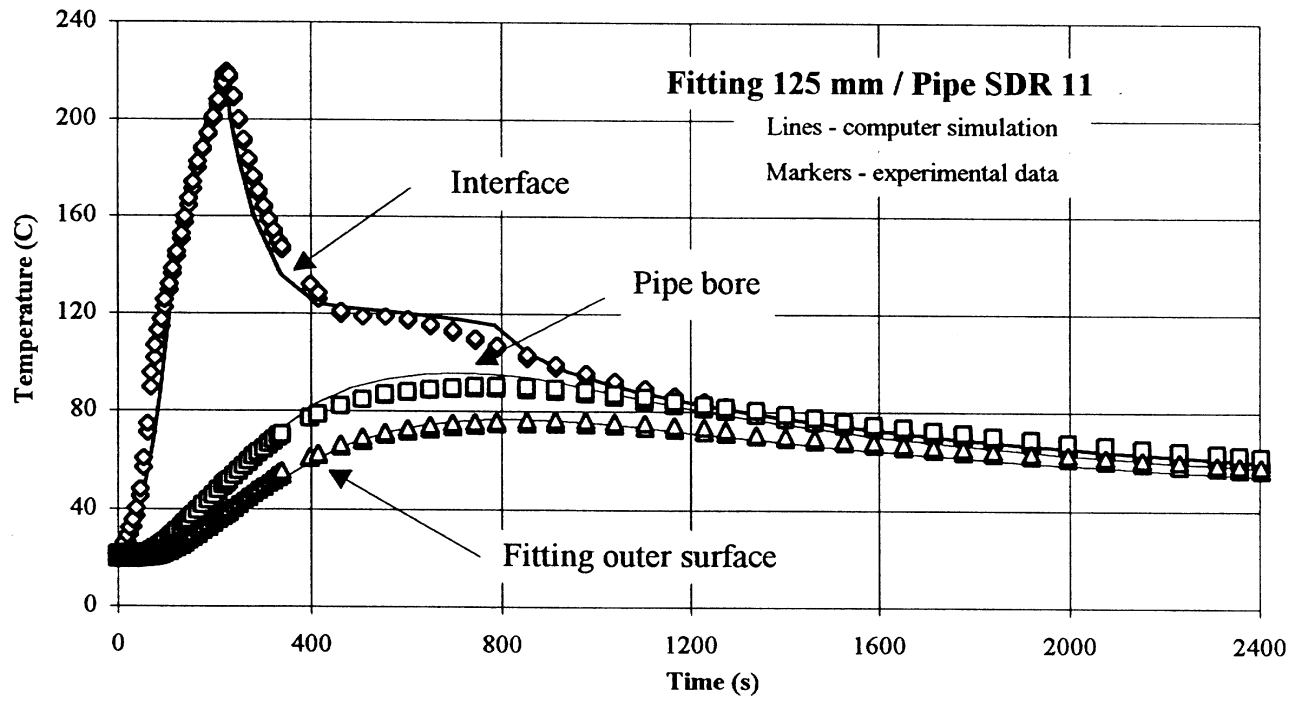

Fig. 8 Correlation between prediction and experimental data (heating-cooling period)

show good agreement with the experimental data, although it is noted that slightly higher temperature gradients and maximum bore temperatures are predicted. The validity of the surface cooling coefficients can be examined by considering the slope of the temperature curves, after the effect of the latent heat release is dissipated (manifested through the plateau that can be seen in the evolution of the interface temperature). The results prove that the implemented values are appropriate.

Concerning the validation of the gap closure prediction, it can be seen from Table 4 that the predicted closure time is slightly higher than the experimental value, but this was expected as the description of the material's coefficient of thermal expansion with temperature was smoothed, to gain computational speed (8). The values in Table 4, as a percentage of the heating time, were identified from Fig. 7. It was shown that this is possible because the gap closure event induces a 'knee' point in the interface time-temperature curve $(\mathbf{8})$.

A good representation of the expansion of the melt into the pipe and fitting domains is given by the temperature distribution across the joint thickness at the end of the heating period. However, the expansion of the melt front continues for a short period of time after the power supply is switched off, as regions of the melt close to the wires are at a much higher temperature than that needed for melting. Thus, to trace the maximum melt expansion, temperature history diagrams at nodes situated through

Table 4 Predicted and measured gap closure times $(\%)$

\begin{tabular}{cc}
\hline Finite element model & Experimental data \\
\hline 42.7 & 37.3 \\
\hline
\end{tabular}

the fitting and pipe thickness were plotted. Nodes on each side of the melt temperature were localized and subsequently the melt front position was computed through interpolation. The values of the maximum melt thickness in the fitting and pipe domains predicted by the model in the centre of the fusion zone are compared with experimental data published by Hinchcliff (11) (from examinations, under cross-polarized light, of thin microtomed sections taken from samples of the fused joints), and good agreement is found (see Table 5).

\section{CONCLUDING REMARKS}

The development and the special features of a finite element electrofusion welding model which allows a full investigation into all the aspects of the process have been presented here. Based on a continuum solid approach, with the molten material treated as an elastic soft solid, the model includes all the basic requirements for accurate modelling, such as temperature-dependent thermal, physical and mechanical material properties, the modelling of the initial fitting-pipe gap and the radial melt movement during the process. The material's specific volume change through melting and solidification was accounted for and implemented into the coefficient of

Table 5 Predicted and measured melt affected zone thickness (mm)

\begin{tabular}{lcc}
\hline & Finite element model* $^{*}$ & Experimental data \\
\hline Fitting & 5.34 & 5.57 \\
Pipe & 3.25 & 3.01 \\
\hline
\end{tabular}

*For the predicted results the melt temperature was taken at the bottom end of the polymer melting range $\left(117-127^{\circ} \mathrm{C}\right)$. 
thermal expansion description, leading to accurate predictions of the gap closure time. An important feature of the model is the ability to simulate the change in the fitting-pipe interface conductance due to the radial melt movement from the fitting to the pipe. This was achieved using an original virtual material movement (VMM) technique and proved to be essential for the validation of the model with respect to temperature history and maximum melt in the joint.

\section{REFERENCES}

1 Mruk, S. A. In Proceedings of Twelfth Plastic Fuel Gas Pipe Symposium, Boston, 1991, pp. 2-22.

2 Saint Royre, D., Gueugnaut, D. and Reveret, D. Test methodology for the determination of optimum fusion welding conditions of PE. J. Appl. Polym. Sci., 1989, 38, 147-162.

3 Pitman, G. L. Electrofusion welding prediction and computer-aided design of fittings. In Proceedings of Sixth Conference on Plastic Pipes, University of York, 1985, pp. 29.1-29.7.

4 Dufour, D. and Meister, E. Polyethylene electrofusion tech- nique: prediction model of welding quality. In Proceedings of International Gas Research Conference, Japan, 1989, pp. 232-243.

5 Nakashiba, A., Nishimura, H., Inoue, F. and Nakagawa, T. Fusion simulation of EF polyethylene joints for gas distribution. Unpublished manuscript, 1992.

6 Nishimura, H., Inoue, F. and Ishikawa, T. Fusion simulation of EF joints. In Proceedings of International Gas Research Conference, 1992, pp. 495-502.

7 O'Donoghue, P. E., Kanninen, M. F., Green, S. T. and Grigory, S. C. Results of a thermomechanical analysis model for EF joining of PE gas pipes. In Proceedings of Twelfth Plastic Fuel Gas Pipe Symposium, Boston, 1991, pp. 331-342.

8 Rosala, G. F. The process mechanics of polymer pipes welding by electro-fusion. $\mathrm{PhD}$ thesis, University of Bradford, 1995.

9 ABAQUS Theory Manual, Version 5.3, Hibbit, Karlsson and Sorensen, Inc., 1994.

10 Technical Information, IMI Scott Limited, 1993.

11 Hinchcliff, F. A. Computer-aided process design and quality prediction in the electrofusion welding of plastic pipes. Universities of Bradford and Brunel, Progress report 8, August 1994. 